\title{
Suicide in older adults: current perspectives
}

This article was published in the following Dove Press journal:

Clinical Interventions in Aging

\author{
Ismael Conejero',2 \\ Emilie Olié ${ }^{-3}$ \\ Philippe Courtet ${ }^{1-3}$ \\ Raffaella Calati ${ }^{1-3}$ \\ 'Institut National de la Santé \\ Et de la Recherche Médicale \\ (INSERM), University of Montpellier, \\ Neuropsychiatry: Epidemiological and \\ Clinical Research, Montpellier, France; \\ ${ }^{2}$ Department of Emergency Psychiatry \\ and Post-Acute Care, Lapeyronie \\ Hospital, Center Hospitalier \\ Universitairere (CHU) Montpellier, \\ Montpellier, France; ${ }^{3}$ FondaMental \\ Foundation, Créteil, France
}

\begin{abstract}
Suicidal behavior in older adults (65 years old and over) is a major public health issue in many countries. Suicide rates increase during the life course and are as high as 48.7/100,000 among older white men in the USA. Specific health conditions and stress factors increase the complexity of the explanatory model for suicide in older adults. A PubMed literature search was performed to identify most recent and representative studies on suicide risk factors in older adults. The aim of our narrative review was to provide a critical evaluation of recent findings concerning specific risk factors for suicidal thoughts and behaviors among older people: psychiatric and neurocognitive disorders, social exclusion, bereavement, cognitive impairment, decision making and cognitive inhibition, physical illnesses, and physical and psychological pain. We also aimed to approach the problem of euthanasia or physician-assisted suicide in older adults. Our main findings emphasize the need to integrate specific stress factors, such as feelings of social disconnectedness, neurocognitive impairment or decision making, as well as chronic physical illnesses and disability in suicide models and in suicide prevention programs in older adults. Furthermore, the chronic care model should be adapted for the treatment of older people with long-term conditions in order to improve the treatment of depressive disorders and the prevention of suicidal thoughts and acts.
\end{abstract}

Keywords: suicide, attempted suicide, older adults, risk factors

\section{Introduction}

Although suicide attempts (SA) are more frequent among adolescents and young adults, ${ }^{1}$ older men and women show the highest suicide rate in almost all countries, ${ }^{2}$ reaching 48.7/100,000 in the USA for white men (more than four times the nation's age-adjusted rate of $11.1 / 100,000)$ and 140/100,000 in rural China for men. ${ }^{3}$ Moreover, the rate of suicide increases with age among people older than $60 .{ }^{4}$ Conversely, several studies have shown a decrease of SA during life course. Hawton and Harriss found that the ratio of deliberate self-harm to suicide varied from 200 among teenagers to 10 in individuals aged 60 and older. ${ }^{5}$ Furthermore, within older adults who died by suicide, SA prevalence decreased with age. ${ }^{6}$ Lifetime SA prevalence was higher in young-olds (65-74 years) compared to middle-olds (75-84 years). In the same study, prevalence of any psychiatric treatment and disorder also decreased with age. The same decrease was found for legal and financial stressors and relationship problems. In contrast, physical conditions increased, as well as bereavement. Fassberg et al reported physical illnesses, such as cancer, neurologic disorder, pain, liver disease, genital disorders, or rheumatoid disorders, along with physical disability as strongly associated with SA in older people. ${ }^{7}$ Furthermore, the same team of researchers found that limited social connectedness is associated with suicidal ideation (SI), non-fatal suicidal behavior, and suicide among older adults. ${ }^{8}$ Finally, Finestone and Blackmer argued that some neurologic diseases, such as stroke, may alter decision-making processes by affecting cognitive capacity and inducing language deficits. ${ }^{9}$ The implications of impaired
Correspondence: Raffaella Calati INSERM, University of Montpellier, Neuropsychiatry: Epidemiological and Clinical Research, University of Montpellier, 39 Avenue Charles Flahault, 34093 Montpellier Cedex 5, France Tel +33 684593630

Email raffaella.calati@gmail.com 
decision making and loss of control in SA were also sustained in qualitative studies, together with feelings of social connectedness and meaninglessness. ${ }^{10}$

Among the risk factors for suicidal behavior, the main risk factors for suicidal death are becoming a widow/ widower, having other mental disorders, physical illness, and bereavement. ${ }^{11}$ Sinyor et al identified three clusters among patients aged 80 and older who died by suicide: the first cluster included married or widowed patients, the second was composed of individuals who were living alone or socially isolated, and the third one included people suffering from dementia or depression. ${ }^{12}$

Hence, the literature shows that specific sociodemographic (social isolation, marital status, bereavement) or clinical (dementia, cognitive impairment, and physical illness) risk factors may be related with suicidal behavior in older adults. In particular, according to the stress-diathesis model of suicidal behavior, a vulnerability or diathesis as a distal risk factor predisposes individuals to suicidal behavior in the presence of specific situational stressors. ${ }^{13}$ The aim of this narrative review is to highlight the main risk factors of suicidal thoughts and behaviors among older adults. We also aim to address the problem of euthanasia or physicianassisted suicide (EAS) in older people.

\section{Methods}

A literature search was performed to identify studies focusing on suicide among older adults. PubMed database was used to search articles using the broad search terms (suicid* OR selfharm) AND (older* OR elderly). Moreover, when needed, we added specific Medical Subject Headings (eg, dementia). Any form of suicidal outcome was considered: suicide, SA, and SI. Studies were included if 1) they examined older adults; 2) they focused on any form of suicidal outcome: suicide, SA, and SI; 3) they were written in English language. The reference lists of the identified studies and reviews were checked as well for further relevant articles.

Our aim was not to perform a systematic review, but to provide an updated picture of current evidence and a critical evaluation of most recent findings. For this, we included the most representative studies. Our criteria for the representativeness of the studies were: recent publication, the impact factor of the publication, the sample size, the authority of the experts in the field, and the type of publication, such as meta-analyses/reviews.

\section{Mental and neurocognitive disorders}

Mental disorders play a consistent role in older adults' suicide, together with physical conditions, functional impairment, and social problematics. ${ }^{14}$ The prescription records of older adults dead by suicide (1992-2000) in Ontario $(n=1,354)$ were examined to investigate 17 illnesses potentially associated with suicide. ${ }^{15}$ Besides physical conditions, depression, bipolar disorder, anxiety disorders, and psychotic disorders seemed to have an impact on suicide. Among these disorders, depression is frequently underdiagnosed and undertreated in older adults. ${ }^{16}$ Anxiety disorders, in comorbidity with depression, play a consistent role as well, since they are involved in one of every six older adults' suicide deaths. ${ }^{17}$ Moreover, in a cohort including patients from both the Veterans Affairs National Patient Care Database and the Centers for Disease Control and Prevention's National Death Index, psychiatric disorders with the strongest associations with suicide were bipolar disorder, depression, substance use disorders (both alcohol and drugs), schizophrenia, and anxiety disorders, including post-traumatic stress disorder (PTSD). ${ }^{18}$ In fact, PTSD was linked to suicide risk in both military and civilian samples. ${ }^{19,20}$ We should underline that, in countries such as the USA, the PTSD issue may be more relevant, given the size of the ex-military population, as well as in the Middle East, in some African states, and in South America.

According to a review of the literature including 40 studies, suicide victims often visit their physician in the month before suicide. ${ }^{21}$ In Europe, Australia, and the USA, $45 \%$ of them will have a contact with their primary care provider within the month before suicide, while only $20 \%$ would have seen a mental health professional in the same period.

Concerning psychotropic drugs, among majorly depressed older adults, a lower rate of response to all classes of antidepressants was found in males, patients of older age, and patients with a longer mean duration of the current episode. ${ }^{22}$ On the contrary, a higher response rate was found in patients with a higher baseline severity and at the first depressive episode. Moreover, lithium anti-suicidal effect, plausibly related to its anti-aggressive effects, was found in older adults living in nursing homes. ${ }^{23}$

Among neurocognitive disorders, dementia represents a risk factor for SI and suicidal behaviors. For example, Alzheimer's disease was associated with a moderate risk of suicide. ${ }^{24}$ Huntington's disease as well was linked to an increased suicide risk. ${ }^{25}$

Another aspect to consider is the personal impact of disclosure of a dementia diagnosis (ie, depression and suicidal tendencies). ${ }^{26,27}$ In fact, an increase in timely diagnosis rates is expected, since 1) the preclinical diagnosis of dementia and Alzheimer's disease, in particular, may become a reality, and 2) the wide dissemination of scientific knowledge about dementia will constantly increase patient education and awareness 
on this issue. The rate of SI and suicidal behaviors among patients who experience a diagnosis of dementia could be higher. However, the disclosure of the diagnosis could have a positive impact as well: spending more time with loved ones, forming partnerships with other people with the diagnosis, receiving therapeutic support, and improving the quality of patient care. ${ }^{28,29}$

Finally, concerning the effects of entering long-term care facilities, the rate of SI seemed to be higher while suicide was rarer. ${ }^{30}$ Reported correlates of suicidal thoughts were: depression, social isolation, loneliness, and functional decline. The organizational-level characteristics of the care facilities seemed to have an impact as well (staff turnover, facility size).

\section{Social exclusion, loneliness, and bereavement}

In his Interpersonal Theory of Suicide, Joiner has emphasized the impact of social disconnection on suicidal thoughts and behaviors. ${ }^{31}$ According to this theory, SI is the result of feelings of thwarted belongingness and perceived burdensomeness. The suicidal act emerges in individuals with an acquired capability to commit suicide as a consequence of repeated negative and painful events. Conversely, strong social support has a protective effect toward late life stressors ${ }^{32}$ and may protect older adults from suicide as well. Older people experience several situations of social stress and adverse social experience, such as retirement, widowing, or lack of new intimate relationships. ${ }^{33,34}$ Their quality of perceived social support is highly associated with global health status. ${ }^{35}$

Harrison et al, in a cross-sectional case-control study, included depressed patients with SI or a history of SA, nonsuicidal depressed participants, and non-depressed participants aged 60 years and older. ${ }^{36}$ Suicidal patients reported the lowest social support, lowest sense of belonging, and the highest level of chronic interpersonal difficulties. In the same line, in a community sample of individuals aged 65 years and older, less perceived social support predicted increased levels of depression and SI. ${ }^{37}$ However, no relationship was found among the sense of belonging, marital status, and SI. The authors suggested that the lack of association between marital status and SI may be due to the composition of the sample, with $79 \%$ of the participants being females. Moreover, sense of belonging has been reported as a protective factor against SI in older people, but with gender differences ${ }^{38}$ In addition, subjective report of social support and perceived belonging to the community - indicators of social integration - are related to suicide. The relationship between social integration indices (family and non-family support, such as the frequency of social contacts, and the number of non-family support members within 1 hour of travel scored with the Duke Social Support Index) and suicidal behavior was evaluated in patients who died by suicide and in living controls aged 50 years and older. People who died by suicide were less likely to be married, have children, or be involved in religious practice. Association between low social integration and suicide remained significant after controlling for mental disorders and employment status. ${ }^{39}$ Family conflicts and employment change are independent risk factors for suicide in adults over 50 years. ${ }^{40}$ Moreover, religious attendance, which is related to social integration and social contact, was negatively associated with current SI, but not with SA in depressed older patients. ${ }^{41}$ According to the authors, the relationship between public religious practice and SI may be mediated by perceived social support.

In addition to the low sense of belongingness, poor social support, and social integration, the death of close relatives is a highly stressful event and constitutes a risk factor for suicide. Mogensen et al reported the highest suicide risk during the 6 months following the loss of a close relative. ${ }^{42,43}$ The highest odds ratios for suicide were found in persons aged 45 and older who had lost a partner within the last month. In the 45-64 years age group, the odds ratios were 4.33 (95\% $\mathrm{CI}=1.24-15.21)$ and $3.46(95 \% \mathrm{CI}=1.77-6.76)$ in the 65 and older age group, respectively. McLaren et al reported an effect of gender underlying SI following widowing in an Australian community sample aged between 65 and $98 .{ }^{43}$ Widowhood was significantly associated with SI in men and women. In addition, in the mediation model, loss of a spouse was associated with lower level of belongingness in men, but not in women. The differential link between bereavement and sense of belongingness between men and women may be due to the different psychosocial function of marriage or lower ability for men to maintain social ties compared with women.

Altogether, these findings support the need to clearly evaluate the social network and support of older adults due to its influence on health outcomes, including SI and suicidal behaviors.

\section{Cognitive impairment, decision making, and cognitive inhibition}

Suicidal vulnerability has been related to cognitive features in the adult middle-aged population, such as impaired decision making (eg, the choice of options with high immediate reward, but disadvantageous in the long term $)^{44,45}$ or altered recognition of emotional stimuli (facial expressions). ${ }^{46}$ Despite the paucity of studies on older adults, recent findings have also shown the implication of neuropsychological 
alterations underlying diathesis for suicidal behavior in the older adults. These alterations mainly involve decision making, executive control, and, more specifically, cognitive inhibition, ${ }^{47}$ which is one of the three subdomains belonging to executive functions along with mental shifting and information updating. ${ }^{48}$

Clark et al have evaluated decision making in 98 subjects aged over 60 years using the Cambridge Gambling Task (assessing decision making and risk-taking behaviors). ${ }^{49}$ According to the authors, decision making involves the evaluation of anticipated positive and negative outcomes and the selection of a behavioral response that maximizes expected value. Results show that quality of decision making was reduced in depressed suicide attempters compared with depressed non-attempters and healthy controls. In addition, altered decision making was associated with poor perceived social problem solving. The authors suggested that impaired decision making in older suicide attempters may be not related to the level of impulsivity, but rather with the lack of capacity to consider prior experiences and probable consequences of their decisions. Furthermore, a recent study did not confirm the presence of poor Iowa Gambling Task (involving probabilistic learning via monetary rewards and punishments) performance in suicidal behavior among older adults, but highlights its presence among violent suicide attempters only. ${ }^{50}$

Similarly, cognitive inhibition (defined as the ability to disengage from information processing or block of irrelevant information for a given task ${ }^{51}$ ) has been evaluated among older adults showing vulnerability for suicide in two studies. Although diagnosis of major depressive episode is associated with cognitive dysfunction, ${ }^{52}$ specific decrease of cognitive inhibition performance has been suggested to underlie suicidal behavior among older adults independent of depressive disorder. In the first study involving 31 depressed patients over 65 years old with and without SA history, neuropsychological assessment found significantly poorer performance on the go/No-Go task (evaluating the patient's ability to perform or repress a motor response after a given stimuli) in suicidal relative to non-suicidal patients. ${ }^{53}$ These results were confirmed in the second study comparing 20 depressed inpatients with recent history of suicidal behavior to 20 inpatients diagnosed with major depressive episodes but no history of SA, and 20 healthy controls. ${ }^{47}$ Recent suicide attempters showed decreased performance in the three domains of cognitive inhibition (ie, access to relevant information, suppression of not relevant information, and restraint over thoughts and actions) described by
Hasher et al. ${ }^{54}$ Deficit in cognitive inhibition in patients with vulnerability for suicidal behavior may be related to inability to inhibit intrusive SI and then emergence of negative affects and ruminations leading to suicidal acts. ${ }^{53,55}$ In parallel to the intrusion of SI, impaired cognitive inhibition may alter the ability to make adequate choices during decision making through inadequate processing of useful information. ${ }^{47}$

Furthermore, suicide has been related to difficulties in social problem solving (ie, the ability to resolve interpersonal problems) and feeling of social isolation not only in the middle-aged, but also in the older adults' population. ${ }^{31,56-58}$ In a meta-analysis examining age-related differences in perception of emotions, older adults had more difficulty in recognizing basic emotions (sadness, disgust, anger, fear, surprise, and happiness) than younger adults ${ }^{59}$ in all modalities (face, voice, and body). Hence, Szanto et al evaluated the link between suicidal behavior and the ability to recognize emotions and social functioning. ${ }^{60}$ The Reading in the Mind test, which assesses ability to discriminate emotions of others, was administered to depressed suicide attempters, depressed patients without SA history, and healthy controls. Social relationships were evaluated through the Social Network Index, which assesses the different types of social relationships in which the patient is involved. Suicide attempters did not have well recognize facial emotions compared to non-psychiatric controls, but no significant difference was found between depressed suicide attempters and the non-suicidal depressed group. These results also suggest a specific link between constricted social networks, impaired social problem solving, and suicide in depressed older adults. The authors reported a significant correlation between emotion recognition and global cognitive performance measured using the Mattis Dementia Rating Scale. ${ }^{60}$ This suggests that emotion recognition deficits among suicide attempters may be tightly linked with more global cognitive dysfunction in older adults.

Finally, several studies evaluated cognitive performances in older suicide attempters and findings pointed out executive dysfunction in this population. In depressed patients with and without a recent history of SA (within 3 months) aged 60 and older, Dombrovski et al assessed both executive functioning and global cognitive performance with the Executive Interview (EXIT25) and the Dementia Rating Scale (DRS), respectively. ${ }^{61}$ Relative to non-recent attempters, recent suicide attempters had significantly decreased performances on the DRS total score and on the memory and attention subscales. They also scored worse on the EXIT25 independently of comorbid physical illness, severity of depression, substance use, or psychotropic medication. The authors 
hypothesized that executive dysfunction may predispose a depressed patient with a physical illness and/or interpersonal loss - frequent stressful circumstances in late life - toward suicidal thoughts and/or behaviors. In another study with the same design, Gujral et al evaluated 83 older depressed suicide attempters with current SI, 43 depressed patients with SI but without SA, 54 depressed patients without any history of suicidal acts or ideation, and 48 healthy controls. ${ }^{62}$ All the participants were aged 60 and older. In comparison to controls, suicidal ideators with and without SA had decreased performance on the EXIT25. All depressed patients had worse DRS total score than healthy controls. The authors suggested that cognitive alterations may be a contributing factor to the emergence of SI. Another study showed that depressed patients admitted immediately after a SA had increasing errors with age in the Trail Making Test, part B (a test of mental sequencing and flexibility) than depressed non-SA attempters. ${ }^{63}$ In contrast, no difference either in attention and concentration performances or in conceptual flexibility or abstract reasoning was found between suicide attempters and non-attempters.

Altogether, these findings highlight the implication of several neurocognitive impairments in the diathesis for older adults' SI and suicidal behaviors. Altered decision making and impaired social emotion recognition underlie decreased ability in problem solving, which may lead to SI. Altered coping strategies due to inadequate choices or altered social functioning may constitute the risk factors for emergence of SI or suicidal acts when facing adverse events such as age-related illnesses or interpersonal loss. In addition, older patients showing decreased executive control and a lack of cognitive inhibition may fail to control intrusive suicide ideas and fail to inhibit emergence of negative affects triggering suicidal behaviors.

Results from studies must be considered in light of some limitations. Studies were cross sectional; so, causal inferences are precluded. Moreover, analyses are based on small samples, and some negative findings may be due to lack of statistical power. Finally, several findings were not replicated (notably for decision making or emotion recognition) and should be confirmed jointly with neuroimaging assessment.

\section{Physical illnesses and physical pain}

As we have already reported, among older adults, physical conditions increased with age. ${ }^{6}$ Results of a recent systematic review ( 65 included studies) on older adults (aged 65 and older) evidenced that suicidal behavior is associated with functional disability and specific physical conditions such as cancer, neurologic disorders, chronic obstructive pulmonary disease (COPD), liver disease, male genital disorders, arthritis/arthrosis, and also pain. ${ }^{7}$ Six of the included studies were qualitative ones. In them, among triggers of suicidal act, frustration with disability, illness, and pain were mainly mentioned, together with loss of autonomy, sense of usefulness, dignity, and pleasure in life. Women more often referred to "not wanting to burden others".

Some studies deserve particular attention. In the USA, older suicide decedents, with and without physical problems as a suicide precipitant, were compared. ${ }^{64}$ Physical problems represented a suicide precipitant in $50 \%$ of the cases. Decedents with physical problems were older and more likely to be depressed and to have disclosed suicide intent. In a large Danish register-based cohort study, the association between 39 physical diseases and death by suicide was examined in older adults (aged 65 years and older) living during 1990-2009 ( $\mathrm{n}=1,849,110) .{ }^{65}$ Increased suicide risk was reported for different types of cancer (lung, gastrointestinal, breast, genital, bladder, lymph node), and for cerebrovascular diseases, heart diseases, COPD, gastrointestinal disease, liver disease, arthritis, osteoporosis, prostate disorders, male genital disorders, epilepsy, cataract, and spinal fracture in comparison to individuals without these diagnoses in the last 3 years.

Among physical conditions, cancer, in its link with suicide, is receiving increasing attention. For example, in a review on suicide risk among patients with genitourinary cancer (specifically prostate, bladder, and kidney cancers, but not testicular cancer), older age mainly represented a greater risk for suicide. ${ }^{66}$

Causal mechanisms of the link between physical diseases and suicide have been reported. ${ }^{65}$ The diagnosis of a severe physical disease (eg, cancer) could represent an acute stressful life event; specific diseases (eg, heart diseases, stroke, and COPD) have been associated with a higher risk of subsequent depression, which could be linked to suicide; some physical conditions may cause chronic pain, which is a well-known risk factor for suicide (for a meta-analysis, see Calati et $\mathrm{al}^{67,68}$ ). Finally, functional impairment could play a further consistent role.

\section{Psychological pain}

More specifically, reasons for attempting suicide were explored in older adults with and without serious physical illness. ${ }^{69}$ In a sample of 101 hospitalized suicide attempters, counterintuitively, SA attributions of somatic distress did not differ in patients with and without serious physical illness. 
Globally, attributions of somatic distress were reported in $34 \%$ of patients, while psychological pain was reported in $60 \%$ of them. In particular, older adults with illness were more likely to report psychological pain as the reason for the SA than the older adults without illness; however, this difference was not reported in older adults of 80 years of age or above. The fact that a difference in reporting psychological pain was detected in different ages could be related to cognitive decline (extremely old patients could have no memories related to the period before the attempt) or simply to the lack of specific reasons reported even if psychological distress was actually present. ${ }^{69}$

It is important to note that the concept of psychological pain ("psychache") does not correspond to the affective component of physical pain. Shneidman defines psychache as an intolerable interoceptive experience that includes shame, guilt, humiliation, loneliness, fear, and anger that could emerge from frustration of essential psychological needs; this construct is highly associated with suicide. ${ }^{70}$ For an updated review of the literature on the association between psychological pain and suicide, see Verrocchio et al. ${ }^{71}$ Furthermore, in a recent meta-analysis performed on 20 studies focused on the link between psychological pain and suicidality, psychological pain was found to be higher in those with a lifetime history and/or current SI or SA compared with those with no SI or SA. ${ }^{72}$ So, the assessment of pain may not be specific, and it may not only be a physical issue. This conclusion is further supported by the observed debated overlap in neural systems of physical and psychological-social pain. ${ }^{73}$ Through the frustration of affiliation need, social pain may be considered as a psychological pain exemplar/subtype. Eisenberger defines "social pain" as the unpleasant experience associated with actual or potential damage to one's sense of social connection or social value (owing to social rejection, exclusion, negative social evaluation, or loss). ${ }^{73}$ Evidence shows that, beyond a lexical overlap, psychological-social pain shares similar neural and biologic patterns of physical pain. ${ }^{73-76}$ If we consider the quite established link between SA in particular and a high sensitivity to negative social signals, ${ }^{77}$ the social component of pain may also be involved.

\section{Euthanasia}

EAS is legal in some countries (the Netherlands, Belgium, Luxembourg, Colombia, Switzerland, Canada, and six USA). ${ }^{78}$ EAS is usually considered for patients with an unbearable suffering without prospect of improvement and without reasonable alternatives. In the Netherlands, the percentage of patients in whom an end-of-life decision had preceded death increased from $39 \%$ in 1990 to $58 \%$ in 2015. ${ }^{79} \mathrm{With}$ an aging population, end-of-life care for older people is increasing in importance. Indeed, older age is often accompanied by multimorbidity. According to Steck et al's review, a typical person who died with assistance would be a well-educated male cancer patient, aged 60-85 years. ${ }^{80}$

With increasing age, people rate their subjective health as lower. ${ }^{81}$ Subjects aged 64 years or older from the Longitudinal Ageing Study Amsterdam were asked at three different times (2001, 2005, and 2008) whether they could imagine requesting their physician to end their life (euthanasia), or imagine asking for a pill to end their life if they became tired of living in the absence of a severe disease (end-oflife pill). The proportion of older people with a positive attitude toward euthanasia and an end-of-life pill increased over time. The number of respondents who could imagine requesting euthanasia from their physician was much higher than the number of respondents who could imagine asking for an end-of-life pill. ${ }^{82}$ Blank et al examined the interest of non-terminally ill, hospitalized older patients (age 60 or older) in EAS to determine the stability of these interests over time. Subjects were interviewed about their interest in EAS in the case of five different unexpected life-threatening events. Up to half of the subjects expressed hypothetical acceptance of EAS depending on the scenario. After 6 months, a subset of patients changed their minds about EAS, most often in the direction of initial acceptance to later rejection. Depressive state was highly associated with EAS acceptance. ${ }^{83}$

Steck et al compared the sociodemographic factors between assisted and unassisted suicides in Swiss population. ${ }^{84}$ The median age of the individuals who died with assistance was 72 years (interquartile range 60-79 years) compared with a median age of 50 years (interquartile range 40-64 years) for the individuals who died by unassisted suicide. Compared with unassisted suicide, those dying by assisted suicide were more likely to be women, to have no religious affiliation, to have tertiary education, and to be widowed and living alone. Individuals dying by assisted suicide were also more likely to live in neighborhoods of higher socioeconomic status and to live in urban areas than those dying by unassisted suicide.

\section{Conclusion}

Our results have several clinical implications. General medical practitioners should be better involved in the prevention of suicidal behavior in older adults. In fact, they are the first to have contacts with older suicidal people because 1) of the higher prevalence of physical illness in older adults and its 
link with suicidal behavior, and 2) a consistent proportion of older adults had contacts with the general practitioner more than with the psychiatric services prior to suicide. ${ }^{85}$ Sense of usefulness, feeling of social disconnectedness, and psychological pain associated with chronic physical illness should be assessed by practitioners who take care of older adults because of their strong association with suicidal behavior. Second, as late-life anxiety disorder, in comorbidity with depression, ${ }^{17}$ is strongly associated with suicide, treatment of these psychiatric conditions should be optimized in older adults. In fact, it has been shown by Voshaar et al that anxious older patients were more medicated and received less psychotherapy than younger patients. ${ }^{17}$ Third, life events and typical aspects of older adults' lives should be considered, such as the loss of a spouse. ${ }^{6}$ In particular, an intervention against the development of complicated grief is recommended in the presence of symptom severity and lack of improvement, functional impairment, treatment-seeking behaviors, hopelessness, and suicidal thoughts or behaviors. ${ }^{86}$ Encountering older adults who have been widowed or bereaved, who are socially isolated, and/or who may have cognitive difficulties should raise a red flag in health consultations. Such preventive measures in older patients facing psychosocial adverse events may have beneficial effects toward prevalence and severity of suicidal acts and should be evaluated in clinical studies. Finally, a large amount of evidence has suggested that the chronic care model developed by Wagner et al in the $1990 \mathrm{~s}^{87}$ should be adapted for the treatment of older patients with depressive disorders. ${ }^{88}$ The chronic care model is based on six related domains of organization (delivery system design, patient-provider relationship, decision support, clinical information system, community resources, and health care organization) and provides coordination of health professionals by case managers. Improving the clinical practice in the older depressed population with long-term conditions may be a means for better preventing suicide.

This narrative review of the literature has the strength of providing an updated picture of the main current evidence on suicide in older adults, covering different aspects in their link with suicide: psychiatric and neurocognitive disorders, social exclusion, loneliness and bereavement, cognitive impairment, decision making and cognitive inhibition, physical illnesses, physical and psychological pain, and EAS. However, limitations should be acknowledged as well: this study does not systematically cover the bulk of studies on suicidal risk in older adults; to achieve this aim, systematic reviews and meta-analyses should be performed on each feature included here.
To summarize, older adults are particularly vulnerable to health problems due to several factors reported in this review: mental and neurocognitive disorders, social isolation, feelings of disconnectedness and loss of relatives, neurocognitive impairment and altered decision making, chronic physical illnesses, and physical and psychological pain. These factors increase the complexity of the classical stress-diathesis model for suicidal behavior. Hence, they should be included in the study models of suicide and taken into account in suicide prevention plans.

\section{Disclosure}

Dr Ismael Conejero received fees for participation in scientific boards from Lundbeck. Dr Emilie Olié received research grants from AstraZeneca, Servier, and Institut UPSA de la Douleur and fees for presentations at congresses from Janssen, Lundbeck, Otsuka, and Servier. Prof Philippe Courtet received research grants from Servier and fees for presentations at congresses or participation in scientific boards from Janssen, Lundbeck, Otsuka, and Servier. Dr Raffaella Calati received a grant from FondaMental Foundation, Créteil, France (2015-2016). The authors report no other conflicts of interest in this work.

\section{References}

1. Conejero I, Lopez-Castroman J, Giner L, Baca-Garcia E. Sociodemographic antecedent validators of suicidal behavior: a review of recent literature. Curr Psychiatry Rep. 2016;18(10):94.

2. World Health Organization; 2017. Available from: http:/www.who. int/mental_health/prevention/suicide/suicideprevent/en/. Accessed September 15, 2017

3. Conwell Y, Thompson C. Suicidal behavior in elders. Psychiatr Clin North Am. 2008;31(2):333-356.

4. Shah A, Bhat R, Zarate-Escudero S, DeLeo D, Erlangsen A. Suicide rates in five-year age-bands after the age of 60 years: the international landscape. Aging Ment Health. 2016;20(2):131-138.

5. Hawton K, Harriss L. How often does deliberate self-harm occur relative to each suicide? A study of variations by gender and age. Suicide Life Threat Behav. 2008;38(6):650-660.

6. Koo YW, Kolves K, De Leo D. Suicide in older adults: differences between the young-old, middle-old, and oldest old. Int Psychogeriatr. 2017;29(8):1297-1306.

7. Fassberg MM, Cheung G, Canetto SS, et al. A systematic review of physical illness, functional disability, and suicidal behaviour among older adults. Aging Ment Health. 2016;20(2):166-194.

8. Fassberg MM, van Orden KA, Duberstein P, et al. A systematic review of social factors and suicidal behavior in older adulthood. Int J Environ Res Public Health. 2012;9(3):722-745.

9. Finestone HM, Blackmer J. Refusal to eat, capacity, and ethics in stroke patients: a report of 3 cases. Arch Phys Med Rehabil. 2007;88(11): 1474-1477.

10. Wand AP, Peisah C, Draper B, Brodaty H. Understanding self-harm in older people: a systematic review of qualitative studies. Aging Ment Health. 2018;22(3):289-298.

11. Bonnewyn A, Shah A, Demyttenaere K. Suicidality and suicide in older people. Rev Clin Gerontol. 2009;19(4):271-294. 
12. Sinyor M, Tan LP, Schaffer A, Gallagher D, Shulman K. Suicide in the oldest old: an observational study and cluster analysis. Int J Geriatr Psychiatry. 2016;31(1):33-40.

13. van Heeringen K. Stress-Diathesis Model of Suicidal Behavior. In: Dwivedi Y, editor. The Neurobiological Basis of Suicide. Boca Raton, FL, USA: CRC Press/Taylor \& Francis; 2012.

14. Conwell Y, Duberstein PR, Caine ED. Risk factors for suicide in later life. Biol Psychiatry. 2002;52(3):193-204.

15. Juurlink DN, Herrmann N, Szalai JP, Kopp A, Redelmeier DA. Medical illness and the risk of suicide in the elderly. Arch Intern Med. 2004;164(11):1179-1184.

16. Allan CE, Valkanova V, Ebmeier KP. Depression in older people is underdiagnosed. Practitioner. 2014;258(1771):19-22.

17. Voshaar RC, van der Veen DC, Kapur N, Hunt I, Williams A, Pachana NA. Suicide in patients suffering from late-life anxiety disorders; a comparison with younger patients. Int Psychogeriatr. 2015; 27(7):1197-1205.

18. Ilgen MA, Bohnert AS, Ignacio RV, et al. Psychiatric diagnoses and risk of suicide in veterans. Arch Gen Psychiatry. 2010;67(11):1152-1158.

19. Panagioti M, Gooding PA, Tarrier N. A meta-analysis of the association between posttraumatic stress disorder and suicidality: the role of comorbid depression. Compr Psychiatry. 2012;53(7):915-930.

20. Krysinska K, Lester D. Post-traumatic stress disorder and suicide risk: a systematic review. Arch Suicide Res. 2010;14(1):1-23.

21. Luoma JB, Martin CE, Pearson JL. Contact with mental health and primary care providers before suicide: a review of the evidence. Am J Psychiatry. 2002;159(6):909-916.

22. Calati R, Salvina Signorelli M, Balestri M, et al. Antidepressants in elderly: metaregression of double-blind, randomized clinical trials. $J$ Affect Disord. 2013;147(1-3):1-8.

23. Muller-Oerlinghausen B, Lewitzka U. Lithium reduces pathological aggression and suicidality: a mini-review. Neuropsychobiology. 2010; 62(1):43-49.

24. Serafini G, Calcagno P, Lester D, Girardi P, Amore M, Pompili M. Suicide risk in Alzheimer's disease: a systematic review. Curr Alzheimer Res. 2016;13(10):1083-1099.

25. Haw C, Harwood D, Hawton K. Dementia and suicidal behavior: a review of the literature. Int Psychogeriatr. 2009;21(3):440-453.

26. Mitchell G, McCollum P, Monaghan C. The personal impact of disclosure of a dementia diagnosis: a thematic review of the literature. Br J Neurosci Nurs. 2013;9(5):223-228.

27. Mattsson N, Brax D, Zetterberg H. To know or not to know: ethical issues related to early diagnosis of Alzheimer's disease. Int J Alzheimers Dis. 2010;2010:841941

28. Mitchell G, McCollum P, Monaghan C. Disclosing a diagnosis of dementia: a background to the phenomenon. Nurs Older People. 2013; 25(10): $16-21$.

29. De Lepeleire J, Buntinx F, Aertgeerts B. Disclosing the diagnosis of dementia: the performance of Flemish general practitioners. Int Psychogeriatr. 2004;16(4):421-428.

30. Mezuk B, Rock A, Lohman MC, Choi M. Suicide risk in long-term care facilities: a systematic review. Int J Geriatr Psychiatry. 2014;29(12): $1198-1211$.

31. Joiner T. Why People Die by Suicide. London: Harvard University Press; 2007.

32. Krause N, Jay G. Stress, social support, and negative interaction in later life. Res Aging. 1991;13(3):333-363.

33. Baumeister RF, Leary MR. The need to belong: desire for interpersonal attachments as a fundamental human motivation. Psychol Bull. 1995;117(3):497-529.

34. Bisconti TL, Bergeman CS. Perceived social control as a mediator of the relationships among social support, psychological well-being, and perceived health. Gerontologist. 1999;39(1):94-103.

35. White AM, Philogene GS, Fine L, Sinha S. Social support and selfreported health status of older adults in the United States. Am J Public Health. 2009;99(10):1872-1878.
36. Harrison KE, Dombrovski AY, Morse JQ, et al. Alone? Perceived social support and chronic interpersonal difficulties in suicidal elders. Int Psychogeriatr. 2010;22(3):445-454.

37. Vanderhorst RK, McLaren S. Social relationships as predictors of depression and suicidal ideation in older adults. Aging Ment Health. 2005;9(6):517-525.

38. McLaren S, Gomez R, Bailey M, Van Der Horst RK. The association of depression and sense of belonging with suicidal ideation among older adults: applicability of resiliency models. Suicide Life Threat Behav. 2007;37(1):89-102.

39. Duberstein PR, Conwell Y, Conner KR, Eberly S, Evinger JS, Caine ED. Poor social integration and suicide: fact or artifact? A case-control study. Psychol Med. 2004;34(7):1331-1337.

40. Duberstein PR, Conwell Y, Conner KR, Eberly S, Caine ED. Suicide at 50 years of age and older: perceived physical illness, family discord and financial strain. Psychol Med. 2004;34(1):137-146.

41. Rushing NC, Corsentino E, Hames JL, Sachs-Ericsson N, Steffens DC. The relationship of religious involvement indicators and social support to current and past suicidality among depressed older adults. Aging Ment Health. 2013;17(3):366-374.

42. Mogensen H, Moller J, Hultin H, Mittendorfer-Rutz E. Death of a close relative and the risk of suicide in Sweden-A Large Scale Register-Based Case-Crossover Study. PLoS One. 2016;11(10):e0164274.

43. McLaren S, Gomez R, Gill P, Chesler J. Marital status and suicidal ideation among Australian older adults: the mediating role of sense of belonging. Int Psychogeriatr. 2015;27(1):145-154.

44. Jollant F, Bellivier F, Leboyer M, et al. Impaired decision making in suicide attempters. Am J Psychiatry. 2005;162(2):304-310

45. Bridge JA, McBee-Strayer SM, Cannon EA, et al. Impaired decision making in adolescent suicide attempters. J Am Acad Child Adolesc Psychiatry. 2012;51(4):394-403.

46. Richard-Devantoy S, Guillaume S, Olie E, Courtet P, Jollant F. Altered explicit recognition of facial disgust associated with predisposition to suicidal behavior but not depression. J Affect Disord. 2013;150(2): 590-593.

47. Richard-Devantoy S, Jollant F, Kefi Z, et al. Deficit of cognitive inhibition in depressed elderly: a neurocognitive marker of suicidal risk. J Affect Disord. 2012;140(2):193-199.

48. Miyake A, Friedman NP, Emerson MJ, Witzki AH, Howerter A, Wager TD. The unity and diversity of executive functions and their contributions to complex "Frontal Lobe" tasks: a latent variable analysis. Cogn Psychol. 2000;41(1):49-100.

49. Clark L, Dombrovski AY, Siegle GJ, et al. Impairment in risk-sensitive decision-making in older suicide attempters with depression. Psychol Aging. 2011;26(2):321-330.

50. Wyart M, Jaussent I, Ritchie K, Abbar M, Jollant F, Courtet P. Iowa gambling task performance in elderly persons with a lifetime history of suicidal acts. Am J Geriatr Psychiatry. 2016;24(5):399-406.

51. Joormann J, Yoon KL, Zetsche U. Cognitive inhibition in depression. Appl Prev Psychol. 2007;12(3):128-139.

52. Elderkin-Thompson V, Mintz J, Haroon E, Lavretsky H, Kumar A. Executive dysfunction and memory in older patients with major and minor depression. Arch Clin Neuropsychol. 2007;22(2):261-270.

53. Richard-Devantoy S, Annweiler C, Le Gall D, Garre JB, Olie JP, Beauchet O. Cognitive inhibition in suicidal depressed elderly: a casecontrol pilot study. J Clin Psychiatry. 2011;72(6):871-872.

54. Hasher L, Zacks RT, May CP. Inhibitory control, circadian arousal and age. Attention and Performance. 1999;17:653-675.

55. Marzuk PM, Hartwell N, Leon AC, Portera L. Executive functioning in depressed patients with suicidal ideation. Acta Psychiatr Scand. 2005; 112(4):294-301.

56. Foster T. Adverse life events proximal to adult suicide: a synthesis of findings from psychological autopsy studies. Arch Suicide Res. 2011; 15(1):1-15.

57. Pollock LR, Williams JM. Problem solving and suicidal behavior. Suicide Life Threat Behav. 1998;28(4):375-387. 
58. Gibbs LM, Dombrovski AY, Morse J, Siegle GJ, Houck PR, Szanto K. When the solution is part of the problem: problem solving in elderly suicide attempters. Int J Geriatr Psychiatry. 2009;24(12):1396-1404.

59. Ruffman T, Henry JD, Livingstone V, Phillips LH. A meta-analytic review of emotion recognition and aging: implications for neuropsychological models of aging. Neurosci Biobehav Rev. 2008;32(4):863-881.

60. Szanto K, Dombrovski AY, Sahakian BJ, et al. Social emotion recognition, social functioning, and attempted suicide in late-life depression. Am J Geriatr Psychiatry. 2012;20(3):257-265.

61. Dombrovski AY, Butters MA, Reynolds CF 3rd, et al. Cognitive performance in suicidal depressed elderly: preliminary report. $\mathrm{Am} J$ Geriatr Psychiatry. 2008;16(2):109-115.

62. Gujral S, Dombrovski AY, Butters M, Clark L, Reynolds CF 3rd, Szanto K. Impaired executive function in contemplated and attempted suicide in late life. Am J Geriatr Psychiatry. Epub 2013 Feb 6.

63. King DA, Conwell Y, Cox C, Henderson RE, Denning DG, Caine ED. A neuropsychological comparison of depressed suicide attempters and nonattempters. J Neuropsychiatry Clin Neurosci. 2000;12(1):64-70.

64. Choi NG, DiNitto DM, Marti CN, Conwell Y. Physical health problems as a late-life suicide precipitant: examination of coroner/medical examiner and law enforcement reports. Gerontologist. Epub 2017 Sep 18.

65. Erlangsen A, Stenager E, Conwell Y. Physical diseases as predictors of suicide in older adults: a nationwide, register-based cohort study. Soc Psychiatry Psychiatr Epidemiol. 2015;50(9):1427-1439.

66. de Lima MM Jr, Tobias-Machado M. Suicide in patients with genitourinary malignancies. Eur J Cancer Care (Engl). 2017;26(6).

67. Calati R, Artero S, Courtet P, Lopez-Castroman J. Framing the impact of physical pain on suicide attempts. A reply to Stubbs. J Psychiatr Res. 2016;72:102-103.

68. Calati R, Laglaoui Bakhiyi C, Artero S, Ilgen M, Courtet P. The impact of physical pain on suicidal thoughts and behaviors: meta-analyses. J Psychiatr Res. 2015;71:16-32.

69. Wiktorsson S, Berg AI, Wilhelmson K, et al. Assessing the role of physical illness in young old and older old suicide attempters. Int $J$ Geriatr Psychiatry. 2016;31(7):771-774.

70. Shneidman ES. Suicide as psychache. J Nerv Ment Dis. 1993;181(3): 145-147.

71. Verrocchio MC, Carrozzino D, Marchetti D, Andreasson K, Fulcheri M, Bech P. Mental pain and suicide: a systematic review of the literature. Front Psychiatry. 2016;7:108.

72. Ducasse D, Holden RR, Boyer L, et al. Psychological pain in suicidality: a meta-analysis. J Clin Psychiatry. Epub 2017 Aug 29.

73. Eisenberger NI. The pain of social disconnection: examining the shared neural underpinnings of physical and social pain. Nat Rev Neurosci. 2012;13(6):421-434.
74. Eisenberger NI. Social pain and the brain: controversies, questions, and where to go from here. Annu Rev Psychol. 2015;66:601-629.

75. Kross E, Berman MG, Mischel W, Smith EE, Wager TD. Social rejection shares somatosensory representations with physical pain. Proc Natl Acad Sci U S A. 2011;108(15):6270-6275.

76. Meyer ML, Williams KD, Eisenberger NI. Why social pain can live on: different neural mechanisms are associated with reliving social and physical pain. PLoS One. 2015;10(6):e0128294.

77. Jollant F, Lawrence NS, Giampietro V, et al. Orbitofrontal cortex response to angry faces in men with histories of suicide attempts. Am J Psychiatry. 2008;165(6):740-748.

78. Emanuel EJ, Onwuteaka-Philipsen BD, Urwin JW, Cohen J. Attitudes and practices of Euthanasia and Physician-Assisted Suicide in the United States, Canada, and Europe. JAMA. 2016;316(1):79-90.

79. van der Heide A, van Delden JJM, Onwuteaka-Philipsen BD. End-oflife decisions in the Netherlands over 25 years. $N$ Engl J Med. 2017; 377(5):492-494

80. Steck N, Egger M, Maessen M, Reisch T, Zwahlen M. Euthanasia and assisted suicide in selected European countries and US states: systematic literature review. Med Care. 2013;51(10):938-944.

81. Gunzelmann T, Hinz A, Brahler E. Subjective health in older people. Psychosoc Med. 2006;3:Doc02.

82. Buiting HM, Deeg DJ, Knol DL, et al. Older peoples' attitudes towards euthanasia and an end-of-life pill in The Netherlands: 2001-2009. J Med Ethics. 2012;38(5):267-273.

83. Blank K, Robison J, Prigerson H, Schwartz HI. Instability of attitudes about euthanasia and physician assisted suicide in depressed older hospitalized patients. Gen Hosp Psychiatry. 2001;23(6):326-332.

84. Steck N, Egger M, Zwahlen M; Swiss National Cohort. Assisted and unassisted suicide in men and women: longitudinal study of the Swiss population. Br J Psychiatry. 2016;208(5):484-490.

85. Cheung G, Merry S, Sundram F. Medical examiner and coroner reports: uses and limitations in the epidemiology and prevention of late-life suicide. Int J Geriatr Psychiatry. 2015;30(8):781-792.

86. Simon NM. Treating complicated grief. JAMA. 2013;310(4) 416-423.

87. Wagner EH, Austin BT, Von Korff M. Organizing care for patients with chronic illness. Milbank Q. 1996;74(4):511-544.

88. McEvoy P, Barnes P. Using the chronic care model to tackle depression among older adults who have long-term physical conditions. J Psychiatr Ment Health Nurs. 2007;14(3):233-238.
Clinical Interventions in Aging

\section{Publish your work in this journal}

Clinical Interventions in Aging is an international, peer-reviewed journal focusing on evidence-based reports on the value or lack thereof of treatments intended to prevent or delay the onset of maladaptive correlates of aging in human beings. This journal is indexed on PubMed Central, MedLine,

\section{Dovepress}

CAS, Scopus and the Elsevier Bibliographic databases. The manuscript management system is completely online and includes a very quick and fair peer-review system, which is all easy to use. Visit http://www.dovepress. com/testimonials.php to read real quotes from published authors. 